\title{
Generation of Photon-Plasmon Quantum States in Nonlinear Hyperbolic Metamaterials
}

\author{
Alexander N. Poddubny, ${ }^{1,2,3}$ Ivan V. Iorsh, ${ }^{1,4}$ and Andrey A. Sukhorukov ${ }^{3}$ \\ ${ }^{1}$ ITMO University, St. Petersburg 197101, Russia \\ ${ }^{2}$ Ioffe Institute, St. Petersburg 194021, Russia \\ ${ }^{3}$ Nonlinear Physics Centre, Research School of Physics and Engineering, Australian National University, \\ Canberra, Australian Capital Territory 2601, Australia \\ ${ }^{4}$ Division of Physics and Applied Physics, Nanyang Technological University, Singapore 637371, Singapore
}

(Received 4 February 2016; published 13 September 2016)

\begin{abstract}
We develop a general theoretical framework of integrated paired photon-plasmon generation through spontaneous wave mixing in nonlinear plasmonic and metamaterial nanostructures, rigorously accounting for material dispersion and losses in the quantum regime through the electromagnetic Green function. We identify photon-plasmon correlations in layered metal-dielectric structures with $70 \%$ internal heralding quantum efficiency and reveal a novel mechanism of broadband generation enhancement due to topological transition in hyperbolic metamaterials.
\end{abstract}

DOI: 10.1103/PhysRevLett.117.123901

Recent pioneering experiments demonstrated the quantum interference between individual photons in nanoscale plasmonic waveguides [1], operating up to the room temperature $[2,3]$. However, the photon generation relied on spontaneous wave mixing in external bulk nonlinear crystals. Further efforts are focused on the incorporation of photon sources in plasmonic and metamaterial structures, which on the one hand can lead to the realization of fully integrated quantum devices and on the other hand can open new opportunities for manipulating the quantum features of emitted photons, for example, through hyperbolic dispersion $[4,5]$.

The integrated photon-plasmon generation has been so far reported from quantum dot [6] and quantum well [7] structures suffering from inhomogeneous broadening and dephasing. A promising alternative approach to achieve coherent photon generation at room temperatures is to employ spontaneous nonlinear wave mixing processes, which are successfully used in conventional dielectric waveguide circuits [8-10]. This route is feasible since plasmonic structures and metamaterials can enhance and precisely tailor nonlinear wave mixing [11-14].

To fully unlock the potential of the nanoscale plasmonic and metamaterial circuitry for integrated quantum state generation through spontaneous wave mixing, it is necessary to accurately model quantum nonlinear interactions in metal-dielectric structures, providing the fundamentals for structure design and simulation of experimental performance. However, the majority of theoretical techniques have been developed for conventional waveguide structures under the conditions of lossless [15] and nondispersive elements or including just a few optical modes [16-23]. Such methods are not suitable for plasmonic circuits, where frequency dispersion and metal losses are significant, and multiple spatial modes should be taken into account to describe photon emission [4,5].
In this Letter, we present a rigorous approach describing entangled photon-plasmon state generation through spontaneous wave mixing in metal-dielectric nanostructures of arbitrarily complex geometry. We derive ready-to-use explicit formulas for the experimentally measurable photon counts and quantum correlations. They are expressed through the classical electromagnetic Green function satisfying Maxwell's equations and fully incorporating material absorption and dispersion characteristics. We first demonstrate an application of our approach to a bilayer metal-dielectric structure and predict photon-plasmon generation with $\gtrsim 70 \%$ internal heralding efficiency. Then, we analyze a multilayer metal-dielectric hyperbolic metamaterial $[4,5]$, where we reveal a new type of photon-pair generation enhancement due to the broadband phase matching at the topological transition.

We consider the generation of a pair of signal and idler photons from a pump wave through spontaneous nonlinear wave mixing inside metal-dielectric structures, as schematically illustrated in Fig. 1(a). The three relevant processes are the generation of the photon pairs, their (linear) propagation and possible absorption in the structure, and their detection. Importantly, spontaneous nonlinear wave mixing realizes a spatially extended coherent source of

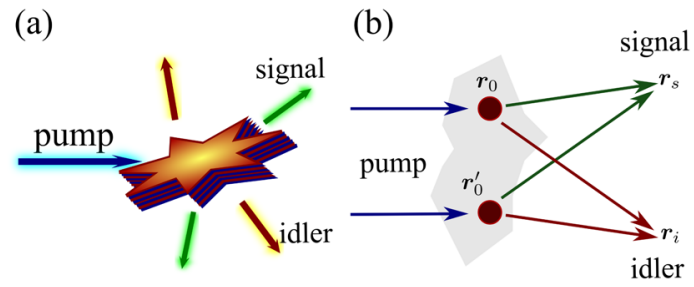

FIG. 1. (a) Scheme of the photon-pair generation from a nonlinear metamaterial. (b) Diagrammatic representation of the two-photon interference according to Eq. (1). 
photon pairs, whose interference can lead to strong quantum entanglement even in the presence of losses [19-21]. This is an important benefit compared to sets of quantum dots [6,7] suffering from dephasing. We consider the weak pumping regime, neglecting the generation of multiple photon pairs. Then, in the presence of linear losses, the output quantum state will be composed of pure photon pairs and single photons in a mixed state [19-21].

The photon-pair generation is described by the Hamiltonian [16] $H_{\mathrm{NL}}=1 / 2 \int d \omega_{1} d \omega_{2}(2 \pi)^{-2} d^{3} r E_{\alpha}^{\dagger} \times$ $\left(\omega_{1}, \boldsymbol{r}\right) E_{\beta}^{\dagger}\left(\omega_{2}, \boldsymbol{r}\right) \Gamma_{\alpha \beta}(\boldsymbol{r})+$ H.c., where $E$ is the electric field operator, $\alpha, \beta=x, y, z$, and $\Gamma_{\alpha \beta}$ is the generation matrix. We consider two possibilities [24], spontaneous parametric down-conversion (SPDC) due to $\chi^{(2)}$ nonlinear susceptibility and spontaneous four-wave mixing (SFWM) governed by $\chi^{(3)}$ nonlinearity, when

$$
\Gamma_{\alpha \beta}(\boldsymbol{r})=\left\{\begin{array}{l}
\chi_{\alpha \beta \gamma}^{(2)}\left(\boldsymbol{r} ; \omega_{1}, \omega_{2} ; \omega_{p}\right) E_{p, \gamma}(\boldsymbol{r}) e^{-i \omega_{p} t} \\
\chi_{\alpha \beta \gamma \delta}^{(3)}\left(\boldsymbol{r} ; \omega_{1}, \omega_{2} ; \omega_{p}, \omega_{p}\right) E_{p, \gamma}(\boldsymbol{r}) E_{p, \delta}(\boldsymbol{r}) e^{-2 i \omega_{p} t} .
\end{array}\right.
$$

$E p$ is the classical pump at frequency $\omega_{p}, \gamma, \delta=x, y, z$.

The linear propagation of the generated photons is governed by the Hamiltonian $H_{\text {lin }}=\int d^{3} r \int_{0}^{\infty} d \omega \hbar \omega \boldsymbol{f}^{\dagger} \cdot \boldsymbol{f}$, where $f_{\alpha}(\boldsymbol{r}, \omega)$ are the canonical bosonic source operators for the quantum electric field [25]: $\boldsymbol{E}(\boldsymbol{r})=\int_{0}^{\infty} d \omega(2 \pi)^{-1} \times$ $\boldsymbol{E}(\boldsymbol{r}, \omega)+$ H.c., $\hat{\boldsymbol{E}}(\omega)=i \sqrt{\hbar} \int d^{3} \boldsymbol{r}^{\prime} G_{\alpha \beta}\left(\boldsymbol{r}, \boldsymbol{r}^{\prime}, \omega\right) \sqrt{\operatorname{Im} \varepsilon\left(\omega, \boldsymbol{r}^{\prime}\right)} \times$ $f_{\beta}\left(\boldsymbol{r}^{\prime}, \omega\right)$. Here, $G$ is the classical electromagnetic Green tensor

$$
\left[\operatorname{rot} \operatorname{rot}-\left(\frac{\omega}{c}\right)^{2} \varepsilon(\omega, \boldsymbol{r})\right] G\left(\boldsymbol{r}, \boldsymbol{r}^{\prime}, \omega\right)=4 \pi\left(\frac{\omega}{c}\right)^{2} \hat{1} \delta\left(\boldsymbol{r}-\boldsymbol{r}^{\prime}\right) .
$$

The advantage of the local source quantization scheme [25] is the possibility to explicitly account for arbitrary strong Ohmic losses and mode dispersion, encoded in the Green function. This method was previously applied [26] to describe the spontaneous two-photon emission [27] from a single atom. However, the current problem is quite distinct from spontaneous two-photon emission because nonlinear spontaneous wave mixing acts as a coherent spatially extended source.

We explicitly introduce the sensors that detect the quantum electromagnetic field [28] to find the experimentally measurable quantities. The sensors are modeled as signal $(s)$ and idler $(i)$ two-level systems with the Hamiltonians $H_{i, s}=\hbar \omega_{i, s} a_{i, s}^{\dagger} a_{i, s}-\hat{\boldsymbol{d}}_{i, s} \cdot \boldsymbol{E}\left(\boldsymbol{r}_{i, s}\right)$, with the resonant energies $\hbar \omega_{s}$ and $\hbar \omega_{i}$, respectively. Here, $a_{s, i}^{\dagger}$ are the corresponding exciton creation and $\hat{\boldsymbol{d}}_{i, s}=a \boldsymbol{d}_{i, s}^{*}+a^{\dagger} \boldsymbol{d}_{i, s}^{*}$ are the dipole momentum operators.

The detected two-quantum state is $|\Psi\rangle=a_{i}^{\dagger} a_{s}^{\dagger}|0\rangle$, with both detectors excited by the photon pair. Formally, the process of photon-pair generation, propagation, and detection can be described by the scattering matrix element $S_{i s}=\langle\Psi|U| 0\rangle$, where $U$ is the evolution operator [29]. We develop a direct perturbation technique [30] and obtain $S_{i s}=-2 \pi i \delta\left(\hbar \omega_{i}+\hbar \omega_{s}-N \hbar \omega_{\text {pump }}\right) T_{i s}$, where $N=1(2)$ for SPDC (SFWM). By construction, the two-photon transition amplitude $T_{i s}$ has the meaning of the complex wave function fully defining the pure two-photon state:

$$
\begin{aligned}
& T_{i s}\left(\boldsymbol{r}_{i}, \omega_{i}, \boldsymbol{d}_{i} ; \boldsymbol{r}_{s}, \omega_{s}, \boldsymbol{d}_{s}\right) \\
& =\sum_{\alpha \beta, \sigma_{i}, \sigma_{s}} d_{i, \sigma_{i}}^{*} d_{s, \sigma_{s}}^{*} \\
& \quad \times \int d^{3} r_{0} G_{\sigma_{i} \alpha}\left(\boldsymbol{r}_{i}, \boldsymbol{r}_{0}, \omega_{i}\right) G_{\sigma_{s} \beta}\left(\boldsymbol{r}_{s}, \boldsymbol{r}_{0}, \omega_{s}\right) \Gamma_{\alpha \beta}\left(\boldsymbol{r}_{0}\right) .
\end{aligned}
$$

This is the central result of our study. The form of Eq. (1) clearly represents the interference between the spatially entangled photons generated in the different points of space $\boldsymbol{r}_{0}$ [41], as schematically illustrated in Fig. 1(b).

The coincidence rate, which defines simultaneous detection of two photons at different positions in space, is found as $W_{i s}=(2 \pi / \hbar) \delta\left(\hbar \omega_{i}+\hbar \omega_{s}-N \hbar \omega_{p}\right)\left|T_{i s}\right|^{2}$. The singlephoton states can be measured by the total count rate of one detector, and for signal photons we obtain [30] $W_{s}\left(\boldsymbol{r}_{s}\right)=$ $(2 / \hbar) \iint d^{3} r_{0}^{\prime} d^{3} r_{0}^{\prime \prime} \sum_{\sigma_{s}, \sigma_{s^{\prime}}} d_{s, \sigma_{s}} d_{s, \sigma_{s^{\prime}}}^{*} \operatorname{Im} G_{\beta \beta^{\prime}}\left(\boldsymbol{r}_{0}^{\prime}, \boldsymbol{r}_{0}^{\prime \prime}, \omega_{p}-\omega_{s}\right)$ $\Gamma_{\alpha \beta}\left(\boldsymbol{r}_{0}^{\prime}\right) \quad \Gamma_{\alpha^{\prime} \beta^{\prime}}^{*}\left(\boldsymbol{r}_{0}^{\prime \prime}\right) \quad G_{\sigma_{s}, \alpha}\left(\boldsymbol{r}_{s}, \boldsymbol{r}_{0}^{\prime}, \omega_{s}\right) \quad G_{\sigma_{s^{\prime}}, \alpha^{\prime}}^{*}\left(\boldsymbol{r}_{s}, \boldsymbol{r}_{0}^{\prime \prime}, \omega_{s}\right)$. We have verified that our general expressions exactly reproduce the previous results for the waveguides with weak losses [19,20]; see Sec. IV D of the Supplemental Material [30].

We now apply the general theory to layered metaldielectric plasmonic structures. First, we analyze the degenerate spontaneous four-wave mixing for the metallic layer of the thickness $d_{\text {silver }}=20 \mathrm{~nm}$ on top of the nonlinear dielectric; see Figs. 2(a)-2(c). Because of the translational symmetry, the total in-plane momentum $\boldsymbol{k}$ of the photons and plasmons is conserved; i.e., $k_{i, \alpha}+k_{s, \alpha}=2 k_{p, \alpha}$ for $\alpha=x, y$. The most interesting situation is realized for oblique pump incidence, giving rise to four different regimes when (a) both signal and idler, (b) only idler, (c) neither signal nor idler, and (d) only signal in-plane wave vectors lie outside the corresponding light cone boundaries $\omega_{i, s} / c$. The first three situations are schematically shown in Figs. 2(a)-2(c). Two-photon generation occurs in case (c), while (b) and (d) correspond to plasmon generation heralded by the far-field photon.

We perform numerical simulations considering isotropic dielectric with electronic $\chi^{(3)}$ nonlinearity tensor as [24] $\chi_{\alpha \beta \gamma \delta}=\chi_{0}\left(\delta_{\alpha \beta} \delta_{\gamma \delta}+\delta_{\alpha \delta} \delta_{\beta \delta}+\delta_{\alpha \gamma} \delta_{\beta \delta}\right)$. We plot in Fig. 2(d) the Fourier transform of the two-photon detection amplitude $\left|T_{i s}\left(\boldsymbol{k}_{s}, z_{i}, z_{s}\right)\right|^{2}$ for $z_{i}=z_{s}=100 \mathrm{~nm}$ above the structure, defined as $T\left(\boldsymbol{k}_{i}\right)=\int d x d y \exp \left(-i k_{x} x-i k_{y} y\right) T(x, y)$, which characterizes the signal-idler generation efficiency in all different regimes. The relevant Fourier transforms of 
(a)

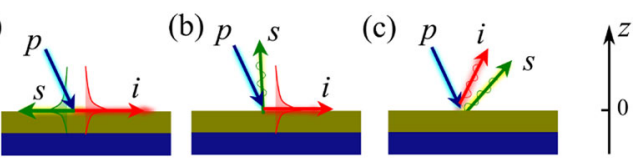

(d)

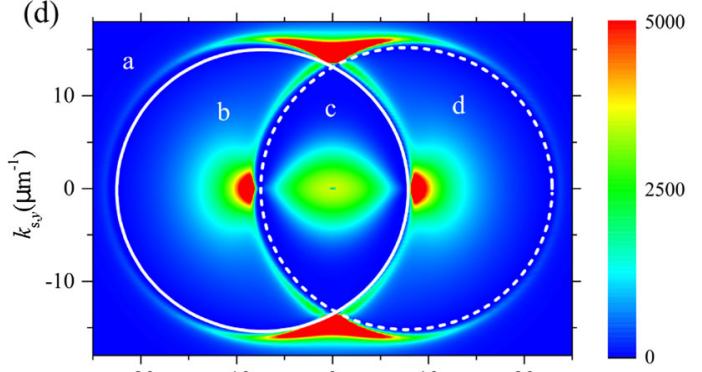

(e)

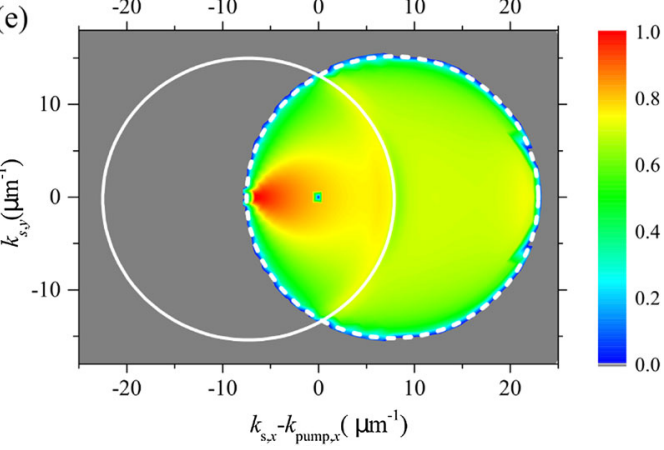

FIG. 2. (a)-(c) Scheme of SFWM generation of a pair of (a) entangled plasmons, (b) entangled photons, and (c) a photon entangled with a plasmon in the gold or nonlinear dielectric structure. (d) Color map of the two-photon detection probability $\left|T\left(\boldsymbol{k}_{i}, \boldsymbol{k}_{s}\right)\right|^{2}$ in the reciprocal space vs the in-plane wave vector components (arbitrary units) in transverse magnetic (TM) polarization $\left(\boldsymbol{d}_{i, s} \propto \boldsymbol{k} \times \boldsymbol{k} \times \hat{\boldsymbol{z}}\right)$ at $z_{i}=z_{s}=100 \mathrm{~nm}$. The signal (solid circle) and idler (dashed circle) light lines are plotted in white. The letters a-d mark the near- and far-field signal and idler generation regimes. (e) Efficiency of signal heralding by far-field idler photons; see Eq. (2). For all plots $\hbar \omega_{i} \approx \hbar \omega_{s} \approx \hbar \omega_{p} \approx 3 \mathrm{eV}$, $\varepsilon_{\text {diel }}=2, \quad d_{\text {silver }}=20 \mathrm{~nm}$, the pump is TM polarized, $k_{p, x}=0.5 \omega_{p} / c$, and silver permittivity is from Ref. [40].

the Green functions were evaluated analytically following Ref. [31]; the details are given in Ref. [30]. Silver permittivity has been taken from Ref. [40] and includes the losses and dispersion. The overall map of the correlations resembles that for the generation of the polarization-entangled photons from a bulk nonlinear uniaxial crystal [42]: it shows strong maxima at the intersections of the signal and idler light cone boundaries. However, contrary to the bulk, the calculated map reflects the two-quantum correlations of both photons and plasmons. In region (c), the shown signal can be directly measured from the far-field photon-photon correlations. For the chosen $30^{\circ}$ pump incidence angle, the bright spot in region (b) of Fig. 2(d) corresponds to the signal photons emitted in the normal direction. The near-field signal in regions (a), (b), and (d) can be recovered by using the grating to outcouple the plasmons to the far field [43] or with the near-field scanning optical microscopy setup [44]. The optimization of the measurement scheme for the specific sample can be handled by the presented general formalism, but it is out of the scope of the current study.

The bright spot in the map in Fig. 2(d) for $k_{s, x}-k_{p, x} \approx$ $10 \mu \mathrm{m}^{-1}$ reveals the resonantly enhanced plasmonic emission heralded by the normally propagating idler photons. The heralding efficiency can be estimated from the comparison of the signal-photon counts $W_{s}$ and the twophoton counts $W_{i s} \propto\left|T_{i s}\right|^{2}$. The details are given in the Supplemental Material [30], and the result reads

$$
Q E=\sum_{z_{i}=-L, L} \sum_{\boldsymbol{d}_{i}=\hat{\boldsymbol{x}} \hat{y}, \hat{z}} \frac{c \cos \theta_{i}}{2 \pi \hbar \omega_{i}} \frac{\left|T_{i s}\left(\boldsymbol{k}_{s}, z_{s}, z_{i}, \boldsymbol{d}_{i}\right)\right|^{2}}{W_{s}\left(\boldsymbol{k}_{s}\right)},
$$

where $\cos \theta_{i}=\sqrt{1-\left(c k_{i} / \omega_{i}\right)^{2}}$. The summation over $z_{i}$ in Eq. (2) accounts for the total idler-photon flux through the surfaces $z_{i}= \pm L$ above and below the nonlinear structure. The calculated values of the signal heralding, shown in Fig. 2(e), are remarkably high. They reach almost $100 \%$ in the case when both signal and idler photons are in the far field; see the bright spot at $k_{s, x}-k_{p, x} \approx-5 \mu \mathrm{m}^{-1}$. In the case of signal plasmons, the heralding efficiency is uniform and about $70 \%$. We note that the results in Fig. 2(e) correspond to the internal heralding efficiency, calculated for the plane pump wave. The external quantum heralding efficiency has to account also for the plasmonic losses due to the propagation from the pump spot to the near-field detector, which can be optimized in the actual experimental setup.

Next, we turn to the multilayered metal-dielectric hyperbolic metamaterial $[4,5]$. This is a strongly anisotropic artificial uniaxial medium, where the effective dielectric constants $\varepsilon_{x x}=\varepsilon_{y y}$ and $\varepsilon_{z z}$ can be of opposite signs, rendering the hyperbolic dispersion law $k_{x}^{2} / \varepsilon_{z z}+k_{z}^{2} / \varepsilon_{x x}=$ $(\omega / c)^{2}$ for the TM polarized waves. In actual calculation, we use the full Green function of the multilayered structure instead of the effective medium approximation [30]. We focus on the nondegenerate SFWM. Enhanced nonlinear processes such as Compton scattering [45] and second harmonic generation $[13,14]$ have been recently predicted in the hyperbolic regime. The photon-pair generation problem is quite different and remains open.

We consider the pump normally incident upon the metamaterial; see Fig. 3(a). Generally, the enhanced local density of states in the hyperbolic metamaterials cannot be harnessed without the special outcoupling of the near field [46]. Here, we avoid this obstacle by considering the nondegenerate spontaneous four-wave mixing when the signal is in the elliptic regime and the idler is in the hyperbolic regime. This allows the signal photons to escape the structure while simultaneously making use of the enhanced density of states due to the hyperbolic plasmons at the idler frequency.

We present in Fig. 3(b) the two-photon correlations $\left|T_{i s}\left(\boldsymbol{k}_{i}, \boldsymbol{k}_{s}\right)\right|^{2}$ in the reciprocal space in the $x y$ plane 
(a)

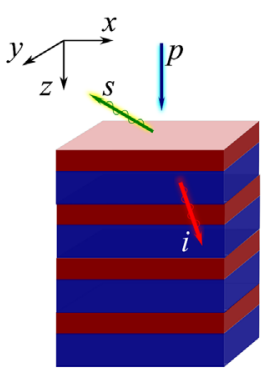

(b)

(c)

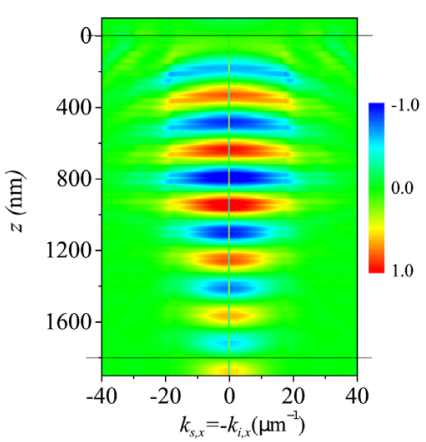

(d)

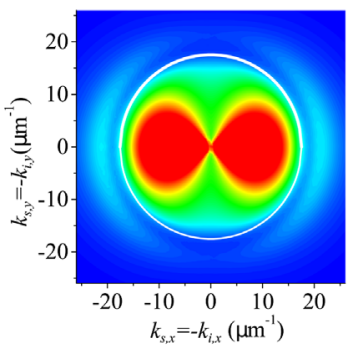

(a)

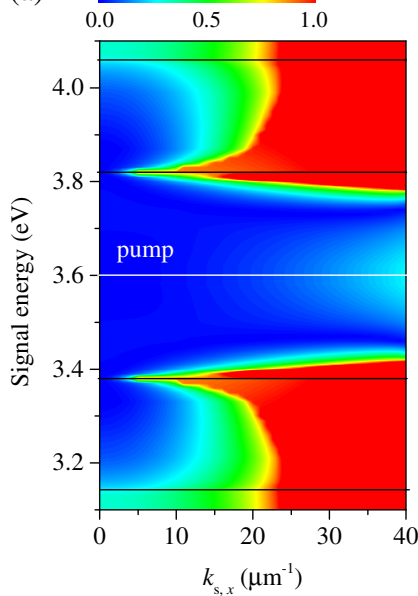

(b)

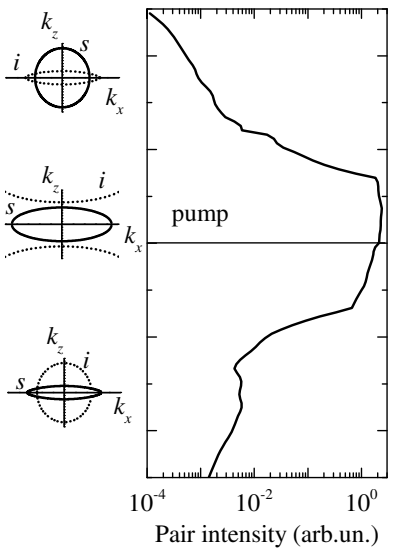

FIG. 4. (a) Phase matching map vs the signal energy and in-plane wave vector. The color corresponds to $\mid \operatorname{Re}\left(k_{s, z}+\right.$ $\left.k_{i, z}-2 k_{\text {pump }, z}\right)\left(d_{1}+d_{2}\right) \mid$. Horizonal black lines show the boundaries between the spectral regions with topologically different dispersion of signal and idler photons (shown in the insets). (b) Photon-pair spectrum in the TM polarization for signal and idler, integrated over the wave vectors inside the signal light cone, calculated for $\hbar \omega_{\text {pump }}=3.6 \mathrm{eV}$ (indicated by a horizontal line) and the same other parameters as in Fig. 3. from a nonlinear hyperbolic metamaterial. (b) Top view of $\left|T_{i s}\left(\boldsymbol{k}_{i}, \boldsymbol{k}_{s}\right)\right|^{2}$ for $z=-100 \mathrm{~nm}$. (c) Side view of $\operatorname{Re} T_{i s}\left(k_{s, x}, z_{i}\right)$. (d) Side view of $\left|T_{i s}\left(k_{s, x}, k_{i, z}\right)\right|^{2}$. Calculated for $\mathrm{TM}$ polarizations of signal and idler detectors, normal pump incidence is polarized along $x, \hbar \omega_{s}=3.46 \mathrm{eV}, \hbar \omega_{p}=3.6 \mathrm{eV}$, $d_{\text {silver }}=12.5 \mathrm{~nm}$, and $d_{\text {diel }}=25 \mathrm{~nm}$.

calculated for both signal and idler at $z_{i}=z_{s}=$ $-100 \mathrm{~nm}$. The structure parameters are similar to that of Ref. [47]; see the caption of Fig. 3. The signal is concentrated in the far-field region inside the light cone. Figure 3(c) shows the side view of the function $\operatorname{Re} T_{i s}\left(k_{s, x}, z\right)$ obtained for signal at $z_{s}=-100 \mathrm{~nm}$ above the structure vs the in-plane wave vector $k_{s, x}$ and idler detection coordinate $z_{s}$. We observe the spatial oscillations of the pattern along the $z$ propagation direction for the hyperbolic idler plasmons within the metamaterial. In order to get an insight of the plasmon propagation, we show in Fig. 3(d) the Fourier transform $\left|T_{i s}\left(k_{s, x}, k_{i, z}\right)\right|^{2}$ as function of the idler-photon wave vector. The solid and dashed white lines show the isofrequency contours at signal and idler frequencies. The maximum of the two-photon response is pinned to the area between the elliptic signal dispersion and the hyperbolic idler dispersion.

To better understand the origin of the enhancement, we analyze in Fig. 4(a) the phase matching conditions in the hyperbolic metamaterial by plotting the map of the momentum mismatch $\left|\operatorname{Re}\left(k_{i, z}+k_{s, z}-2 k_{\text {pump }, z}\right)\right|$ vs the signal energy and the in-plane wave vector. The map is symmetric with respect to the pump energy $\hbar \omega_{p}=3.6 \mathrm{eV}$ (white horizontal line), corresponding to degenerate SFWM. The phase mismatch exhibits dramatic changes

when either signal or idler undergoes topological transition [47] from the elliptic regime to the type-I hyperbolic one (black lines). The intermediate area for $3.4 \mathrm{eV} \lesssim \hbar \omega_{s} \lesssim$ $3.8 \mathrm{eV}$ corresponds to the phase matching realized in the broadband of in-plane wave vectors $k_{x}$ and frequencies. The origin of the broadband phase matching is that the curvatures of the isofrequency contours $d^{2} k_{z} / d k_{x}^{2}$ are of opposite signs at the different sides of the topological transition where $\varepsilon_{z z}$ changes sign. Hence, the major angledependent contributions of signal and idler waves to the phase mismatch cancel each other near the transition frequency. Figure 4(d) shows the spectrum of the integrated two-photon response [Fig. 3(c)] over the in-plane wave vector as function of the signal-photon frequency. We observe a broadband increase in the spectral range $3.4-3.8 \mathrm{eV}$ when the phase matching is realized.

Finally, we note that our general result Eq. (1) reveals an important quantum-classical correspondence for arbitrary structures with quadratic nonlinearity between the photonpair generation through SPDC and sum-frequency generation with classical signal and idler waves, which propagate in the opposite direction to the emulated signal and idler photons. Namely, the far-field sum frequency signal $E_{\mathrm{NL}, \gamma}\left(\boldsymbol{k}_{\mathrm{NL}}, \omega_{i}+\omega_{s}\right)$ is linked to the incident plane waves $\boldsymbol{E}_{s} e^{i \boldsymbol{k}_{s} \boldsymbol{r}-i \omega_{s} t}, \boldsymbol{E}_{i} e^{i \boldsymbol{k}_{i} \boldsymbol{r}-i \omega_{i} t}$ as $E_{\mathrm{NL}, \gamma}=E_{s, \alpha} E_{i, \beta} T\left(\alpha,-\boldsymbol{k}_{i} ; \beta,-\boldsymbol{k}_{s}\right)$, where $T\left(\alpha,-\boldsymbol{k}_{i} ; \beta,-\boldsymbol{k}_{s}\right)$ is the Fourier component of Eq. (1) evaluated for $\boldsymbol{d}_{i}=\boldsymbol{e}_{\alpha}, \boldsymbol{d}_{s}=\boldsymbol{e}_{\beta}$, and $\boldsymbol{E}_{p}=\boldsymbol{e}_{\gamma} e^{-i \boldsymbol{k}_{\mathrm{NL}} \boldsymbol{r}-i\left(\omega_{i}+\omega_{s}\right) t}$. The direction reversal was not considered previously, as only homogeneous lossy waveguides were analyzed [21]. 
Because of the Lorentz reciprocity, the correspondence can be generalized to arbitrary reciprocal waves. This result will be reported in detail separately.

In conclusion, we developed a general theory for the generation of photon and plasmon quantum pairs through spontaneous nonlinear wave mixing, applicable to any structure geometry and accounting for material dispersion and losses through the electromagnetic Green function. We further predicted high internal heralding quantum efficiency and revealed topologically enhanced phase matching in layered metal-dielectric structures. This indicates the experimental feasibility in the presence of metallic losses and suggests even higher performance for all dielectric nonlinear metamaterials and metasurfaces [48-50]. Moreover, our results can extend to other fields, including spontaneous four-wave mixing in Bose-Einstein condensates loaded in tailored potentials [51].

We acknowledge stimulating discussions with A. V. Poshakinskiy, E. L. Ivchenko, S. Saravi, M. M. Glazov, A. S. Solntsev, and M. Steel. This work was supported by the Australian Research Council (Discovery Project DP160100619), the Russian Foundation for Basic Research, and the "Dynasty" Foundation. A. P. acknowledges the support of the Grant of the President of Russian Federation MK-8500.2016.2. I. I. appreciates the support of the Ministry of Education and Science of the Russian Federation (Zadanie No. 3.1231.2014/K), Grant of the President of Russian Federation (MK-5220.2015.2), and Russian Foundation for Basic Research mol-a-dc 16-32-60123.

[1] R. W. Heeres, L. P. Kouwenhoven, and V. Zwiller, Nat. Nanotechnol. 8, 719 (2013).

[2] J. S. Fakonas, H. Lee, Y. A. Kelaita, and H. A. Atwater, Nat. Photonics 8, 317 (2014).

[3] J. S. Fakonas, A. Mitskovets, and H. A. Atwater, New J. Phys. 17, 023002 (2015).

[4] A. Poddubny, I. Iorsh, P. Belov, and Y. Kivshar, Nat. Photonics 7, 948 (2013).

[5] L. Ferrari, C. H. Wu, D. Lepage, X. Zhang, and Z. W. Liu, Prog. Quantum Electron. 40, 1 (2015).

[6] A. Dousse, J. Suffczynski, A. Beveratos, O. Krebs, A. Lemaitre, I. Sagnes, J. Bloch, P. Voisin, and P. Senellart, Nature (London) 466, 217 (2010).

[7] A. Nevet, N. Berkovitch, A. Hayat, P. Ginzburg, S. Ginzach, O. Sorias, and M. Orenstein, Nano Lett. 10, 1848 (2010).

[8] J. W. Silverstone, D. Bonneau, K. Ohira, N. Suzuki, H. Yoshida, N. Iizuka, M. Ezaki, C. M. Natarajan, M. G. Tanner, R. H. Hadfield, V. Zwiller, G. D. Marshall, J. G. Rarity, J. L. O'Brien, and M. G. Thompson, Nat. Photonics 8, 104 (2014).

[9] H. Jin, F. M. Liu, P. Xu, J. L. Xia, M. L. Zhong, Y. Yuan, J. W. Zhou, Y. X. Gong, W. Wang, and S. N. Zhu, Phys. Rev. Lett. 113, 103601 (2014).
[10] A. S. Solntsev, F. Setzpfandt, A. S. Clark, C. W. Wu, M. J. Collins, C. L. Xiong, A. Schreiber, F. Katzschmann, F. Eilenberger, R. Schiek, W. Sohler, A. Mitchell, C. Silberhorn, B. J. Eggleton, T. Pertsch, A. A. Sukhorukov, D. N. Neshev, and Y. S. Kivshar, Phys. Rev. X 4, 031007 (2014).

[11] A. K. Popov and V. M. Shalaev, Appl. Phys. B 84, 131 (2006).

[12] H. Suchowski, K. O’Brien, Z. J. Wong, A. Salandrino, X. B. Yin, and X. Zhang, Science 342, 1223 (2013).

[13] D. de Ceglia, M. A. Vincenti, S. Campione, F. Capolino, J. W. Haus, and M. Scalora, Phys. Rev. B 89, 075123 (2014).

[14] C. Duncan, L. Perret, S. Palomba, M. Lapine, B. T. Kuhlmey, and C. M. de Sterke, Sci. Rep. 5, 8983 (2015).

[15] Z. S. Yang, M. Liscidini, and J. E. Sipe, Phys. Rev. A 77, 033808 (2008).

[16] P. D. Drummond and M. S. Hillery, The Quantum Theory of Nonlinear Optics (Cambridge University Press, Cambridge, 2013).

[17] J. A. Crosse and S. Scheel, Phys. Rev. A 83, 023815 (2011).

[18] M. Kamandar Dezfouli, M. M. Dignam, M. J. Steel, and J. E. Sipe, Phys. Rev. A 90, 043832 (2014).

[19] D. A. Antonosyan, A. S. Solntsev, and A. A. Sukhorukov, Phys. Rev. A 90, 043845 (2014).

[20] L. G. Helt, M. J. Steel, and J. E. Sipe, New J. Phys. 17, 013055 (2015).

[21] L. G. Helt and M. J. Steel, Opt. Lett. 40, 1460 (2015).

[22] T. Onodera, M. Liscidini, J. E. Sipe, and L. G. Helt, Phys. Rev. A 93, 043837 (2016).

[23] M. Kamandar and M. M. Dignam, in CLEO: 2015, OSA Technical Digest (Optical Society of America, Washington, 2015), paper JTu5A.12.

[24] R. W. Boyd, Nonlinear Optics, 3rd ed. (Academic Press, San Diego, 2008).

[25] W. Vogel and D.-G. Welsch, Quantum Optics, 3rd ed. (Wiley, Weinheim, 2006).

[26] A. N. Poddubny, P. Ginzburg, P. A. Belov, A. V. Zayats, and Y. S. Kivshar, Phys. Rev. A 86, 033826 (2012).

[27] A. Hayat, P. Ginzburg, and M. Orenstein, Nat. Photonics 2, 238 (2008).

[28] E. del Valle, A. Gonzalez-Tudela, F. P. Laussy, C. Tejedor, and M. J. Hartmann, Phys. Rev. Lett. 109, 183601 (2012).

[29] C. Cohen-Tannoudji, J. Dupont-Roc, and G. Grynberg, Atom-Photon Interactions: Basic Processes and Applications (Wiley-VCH, New York, 1998).

[30] See Supplemental Material at http://link.aps.org/ supplemental/10.1103/PhysRevLett.117.123901, which includes Refs. [31-40], for the details of derivation.

[31] M. S. Tomas, Phys. Rev. A 51, 2545 (1995).

[32] L. Landau, E. Lifshits, and L. Pitaevskiǔ, Statistical Physics, Part 2, Course of Theoretical Physics (ButterworthHeinemann, Washington, DC, 1980).

[33] S. M. Barnett, B. Huttner, R. Loudon, and R. Matloob, J. Phys. B 29, 3763 (1996).

[34] M. Glazov, E. Ivchenko, A. Poddubny, and G. Khitrova, Phys. Solid State 53, 1753 (2011).

[35] E. Lifshitz and L. Pitaevskiǔ, Physical Kinetics, Course of Theoretical Physics (Butterworth-Heinemann, Washington, DC, 1981). 
[36] H. Haug and A. Jauho, Quantum Kinetics in Transport and Optics of Semiconductors, Springer Series in Solid-State Sciences (Springer, New York, 2007).

[37] M. O. Scully and M.S. Zubairy, Quantum Optics (Cambridge University Press, Cambridge, 1997).

[38] H. Carmichael, An Open Systems Approach to Quantum Optics (Springer, New York, 1993).

[39] L. Landau and E. Lifshits, Statistical Physics, Course of Theoretical Physics Vol. 1 (Butterworth-Heinemann, Washington, DC, 1980).

[40] P. B. Johnson and R. W. Christy, Phys. Rev. B 6, 4370 (1972).

[41] R. Ghosh and L. Mandel, Phys. Rev. Lett. 59, 1903 (1987).

[42] P. G. Kwiat, K. Mattle, H. Weinfurter, A. Zeilinger, A. V. Sergienko, and Y. H. Shih, Phys. Rev. Lett. 75, 4337 (1995).

[43] G. Di Martino, Y. Sonnefraud, S. Kena-Cohen, M. Tame, S. K. Ozdemir, M. S. Kim, and S. A. Maier, Nano Lett. 12, 2504 (2012).
[44] B. le Feber, N. Rotenberg, D. M. Beggs, and L. Kuipers, Nat. Photonics 8, 43 (2014).

[45] I. V. Iorsh, A. N. Poddubny, P. Ginzburg, P. A. Belov, and Y. S. Kivshar, Phys. Rev. Lett. 114, 185501 (2015).

[46] D. Lu, J. J. Kan, E. E. Fullerton, and Z. W. Liu, Nat. Nanotechnol. 9, 48 (2014).

[47] H. N. S. Krishnamoorthy, Z. Jacob, E. Narimanov, I. Kretzschmar, and V. M. Menon, Science 336, 205 (2012).

[48] M. R. Shcherbakov, D. N. Neshev, B. Hopkins, A. S. Shorokhov, I. Staude, E. V. Melik-Gaykazyan, M. Decker, A. A. Ezhov, A. E. Miroshnichenko, I. Brener, A. A. Fedyanin, and Y. S. Kivshar, Nano Lett. 14, 6488 (2014).

[49] S. Makarov, S. Kudryashov, I. Mukhin, A. Mozharov, V. Milichko, A. Krasnok, and P. Belov, Nano Lett. 15, 6187 (2015).

[50] S. Jahani and Z. Jacob, Nat. Nanotechnol. 11, 23 (2016).

[51] R. J. Lewis-Swan and K. V. Kheruntsyan, Nat. Commun. 5, 3752 (2014). 